\section{Esofagitis eosinofílica: diagnóstico y manejo}

\author{
MARÍA JESÚS BALLART ${ }^{1, a}$, HUGO MONRROY ${ }^{1,2,3,4}$, \\ MIRENTXU IRURETAGOYENA ${ }^{5}$, ALEJANDRA PARADA $^{6, b}$, \\ JAVIERA TORRES ${ }^{7}$, ALBERTO ESPINO $^{1,2,3}$
}

\section{Diagnosis and management of eosinophilic esophagitis}

Eosinophilic esophagitis (EoE) is a chronic, immune-mediated disease, induced by food allergens, clinically characterized by symptoms of esophageal dysfunction. Pathologically there is a predominant eosinophilic inflammation. This disease is relatively new, and its definitions have evolved over time. Its prevalence and incidence are increasing and causes clinical problems both in children and adults. Its symptoms include food impaction, dysphagia, symptoms that resemble gastroesophageal reflux, abdominal pain, and vomiting. It can also have extra-digestive symptoms such as rhinosinusitis, chronic cough, recurrent croup and hoarseness. EoE can be associated with other atopic conditions, such as asthma, eczema and food allergies. The diagnosis is made by the analysis of endoscopic biopsies ( $>15$ eosinophils per high power field). Proton pump inhibitors (PPIs) are currently accepted as a treatment for EoE. The clinical and pathological improvement with the use PPIs ceased to be a criterion to define Esophageal eosinophilia responsive to PPIs as a differential diagnosis, since this condition is currently considered within the EoE spectrum. There are three main treatment approaches for EoE: diet, drugs and dilation. Its diagnosis and early treatment are key to avoid or delay its complications, such as stenosis and severe esophageal dysfunction.

(Rev Med Chile 2020; 148: 831-841)

Key words: Eosinophilic Esophagitis; Food Hypersensitivity; Gastroesophageal Reflux; Proton Pump Inhibitors.
'Escuela Medicina, Pontificia Universidad Católica de Chile.

Santiago, Chile.

${ }^{2}$ Departamento de

Gastroenterología, Pontificia

Universidad Católica de Chile.

Santiago, Chile.

${ }^{3}$ Unidad de Endoscopía. Hospital

Clínico UC-Christus. Santiago,

Chile.

${ }^{4}$ Laboratorio de Fisiología

Digestiva. Hospital Clínico UC

Christus. Santiago, Chile.

${ }^{5}$ Laboratorio Clínico, Clínica

Alemana de Santiago, Facultad

de Medicina, Universidad del

Desarrollo. Santiago, Chile.

${ }^{6}$ Departamento Nutrición,

Diabetes y Metabolismo,

Pontificia Universidad Católica de

Chile. Santiago, Chile.

${ }^{7}$ Departamento Anatomía

Patológica, Pontificia Universidad

Católica de Chile. Santiago, Chile.

aEstudiante de Medicina.

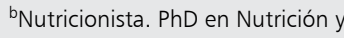
Alimentos.

Trabajo no recibió financiamiento. Los autores declaran no tener conflictos de interés.

Recibido el 25 de junio de 2019, aceptado el 17 de enero de 2020

Correspondencia a: Dr. Alberto Espino Departamento de Gastroenterología. Pontificia Universidad Católica de Chile. Diagonal Paraguay 362. 4to piso. Santiago, Chile. aoespino@uc.cl
L

os eosinófilos están fisiológicamente presentes en la mayoría del tracto gastrointestinal (GI), sin embargo, el epitelio esofágico está desprovisto de eosinófilos en estado sano. La presencia de eosinófilos en el epitelio escamoso esofágico es anormal con un amplio diagnóstico diferencial (Tabla 1), destacando la enfermedad por reflujo gastroesofágico (ERGE) y esofagitis eosinofílica $(\mathrm{EoE})^{1,2}$. La EoE es un diagnóstico relativamente reciente, previamente confundido con $\mathrm{ERGE}^{3}$. Su incidencia y prevalencia han aumentado especialmente en Occidente ${ }^{4}$. Es una causa significativa de morbilidad en población adulta y pediátrica, con impacto en la calidad de vida ${ }^{5}$. Esta revisión tiene por objetivo analizar su epidemiología, fisiopatología, presentación clínica, criterios diagnósticos, tratamiento y pronóstico.

\section{Definiciones}

La EoE es una enfermedad inflamatoria crónica, inmunomediada inducida por alérgenos alimentarios, caracterizada por síntomas relacionados con la disfunción esofágica, tales como impactación alimentaria y disfagia, hallaz- 
Tabla 1. Enfermedades asociadas a eosinofilia esofágica ${ }^{1,7,13}$

\begin{tabular}{l} 
- Esofagitis eosinofílica \\
- Enfermedad por reflujo gastroesofágico \\
- Enfermedades gastrointestinales eosinofílicas \\
- Acalasia \\
- Síndrome hipereosinofílico \\
- Enfermedad de Crohn esofágica \\
- Enfermedad celíaca \\
- Infecciones (fúngicas, virales) \\
- Enfermedades del tejido conectivo \\
- Desórdenes autoinmunes \\
- Vasculitis \\
- Reacciones de hipersensibilidad a drogas \\
- Esofagitis inducida por píldoras \\
- Enfermedad de injerto vs huésped \\
- Síndrome de Marfan tipo II \\
- Síndrome hiper-lgE \\
- Síndrome de tumor hamartoma PTEN (proteína tirosina \\
fosfatasa) \\
- Síndrome de Netherton \\
- Síndrome de atopía grave y desgaste metabólico \\
\hline
\end{tabular}

gos endoscópicos sugerentes y en la histología, eosinofilia esofágica mayor a 15 eosinófilos por campo de aumento mayor (eos/hpf) ${ }^{2,6}$. Según la guía del American College of Gastroenterology (ACG) de 20137, es fundamental excluir otras causas de eosinofilia y que se observe eosinofilia en la mucosa esofágica que persista posterior a una prueba terapéutica con inhibidores de la bomba de protones (IBP). Sin embargo, estos criterios asumen que solo ERGE puede responder a IBP y que son entidades mutuamente excluyentes, sin existir una sobreposición entre ambas. También se incorporó el concepto de "eosinofilia esofágica respondedora a IBP” (PPI-REE, por sus siglas en inglés), sin embargo, estudios prospectivos no han encontrado diferencias en la presencia de fenómenos atópicos, test de alergias, test de monitorización de $\mathrm{pH}$, scores endoscópicos entre PPI-REE y pacientes tradicionalmente diagnosticados con EoE, dificultando distinguir entre ambas y poder predecir la respuesta a IBP ${ }^{8-10}$. Un estudio evaluó la respuesta a IBP en 60 pacientes con EoE documentada, concluyendo que más de $50 \%$ responde a la prueba terapéutica de IBP con doble dosis por 8 semanas ${ }^{11}$. Una revisión japonesa comparó la clínica, hallazgos endoscópicos, histopatología y expresión de genes entre pacientes con EoE y PPI-REE, encontrando que no había diferencias y que ambas representarían la misma condición o variaciones de una misma enfermedad, constituyendo así PPI-REE un subtipo de EoE ${ }^{12}$. Las guías clínicas más recientes han actualizado los criterios diagnósticos para EoE, resolviendo eliminar el criterio del requerimiento de la prueba terapéutica con IBP para poder diagnosticar $\mathrm{EoE}^{13,14}$. En conclusión, actualmente los IBP son considerados como opción terapéutica de EoE y no un criterio diagnóstico, ya que reducen los síntomas y eosinofilia en esófago, con varios mecanismos que explicarían su efecto, además de la supresión de ácido gástrico, como efectos antiinflamatorios y función de barrera.

\section{Epidemiología}

La incidencia y prevalencia de EoE va en aumento, especialmente en los países occidentales $^{15}$. En Chile y Latinoamérica se desconoce su epidemiología debido a la ausencia de estudios poblacionales, y solo se reportan series con pocos casos $^{15-19}$. La prevalencia de EoE se estima en 0,5 a 1 por 1.000 personas en países occidentales. No está claro si el aumento en el diagnóstico de EoE realmente representa una incidencia creciente o un aumento del diagnóstico dirigido o ambos ${ }^{15,20,21}$. Aproximadamente 2 a $7 \%$ de los pacientes adultos sometidos a endoscopía digestiva alta (EDA) con biopsias tienen criterios de $\mathrm{EoE}^{22,23}$, lo que aumenta a $22 \%$ si la disfagia es la indicación de la $\mathrm{EDA}^{24}$. Más de un tercio de los adultos que presentan impactación alimentaria tiene EoE como diagnóstico subyacente ${ }^{25}$. La edad media al diagnóstico varía de 6 a 12 años en niños y 30 años en adultos. Los hombres son 3 a 4 veces más afectados que las mujeres. La EoE es más común en pacientes caucásicos y tiene una fuerte asociación con afecciones atópicas como asma, eccema, rinitis $\mathrm{y}$ alergias alimentarias ${ }^{1,26}$.

\section{Fisiopatología}

Factores genéticos, ambientales y alérgenos estarían implicados en la patogénesis de la EoE. La patogenia se comprende parcialmente: la EoE 
sería secundaria a una respuesta inmune mediada por las células T helper 2 (Th2) y no IgE mediada ${ }^{27}$, similar a otras condiciones alérgicas. Los alérgenos alimentarios inducirían a las células Th2 a producir IL-13, causando la sobreexpresión de eotaxina-3 y periostina, además de la regulación negativa de filagrina y desmogleína 1. La eotaxina-3 es un quimioatrayente de eosinófilos responsable de la acumulación de ellos en la EoE. La regulación negativa de filagrina y desmogleína 1 contribuye a una función de barrera deteriorada. Las células Th2 activadas también producen IL-5, que es responsable de la proliferación y maduración de los eosinófilos. Además de los eosinófilos, se ha demostrado que los mastocitos, los basófilos y las células iNKT contribuyen a la patogénesis de la EoE. Los mastocitos promueven la inflamación y la fibrosis a través de la producción de histamina y TGF- $\beta^{1,6,28}$.

\section{Clínica}

Según una revisión sistemática ${ }^{26}$, los síntomas más comunes en niños fueron vómitos, dolor abdominal, disfagia e impactación alimentaria (Tabla 2), mientras que en adultos fueron disfagia, impactación alimentaria, pirosis, dolor torácico y regurgitación ácida (Tabla 3). Entre 28 y 86\% de

Tabla 2. Síntomas comunes de EoE según frecuencia en niños ${ }^{26}$

\begin{tabular}{|lc|}
\hline Síntoma & Frecuencia (\%) \\
\hline Vómitos & $16,7-59,6 \%$ \\
\hline Dolor abdominal & $15,7-56,6 \%$ \\
\hline Disfagia & $4,8-60,9 \%$ \\
\hline Impactación alimentaria & $6,7-21,7 \%$ \\
\hline
\end{tabular}

Tabla 3. Síntomas comunes de EoE según frecuencia en adultos ${ }^{26}$

\begin{tabular}{|lc|}
\hline Síntoma & Frecuencia (\%) \\
\hline Disfagia & $46,2-94,5 \%$ \\
\hline Impactación alimentaria & $16,9-65,7 \%$ \\
\hline Pirosis & $7,7-54,5 \%$ \\
\hline Dolor torácico & $0-35,5 \%$ \\
\hline Regurgitación ácida & $4,5-38,2 \%$ \\
\hline
\end{tabular}

adultos y 42 y $93 \%$ de niños con EoE tienen otra enfermedad alérgica concurrente, incluyendo alergias alimentarias, rinitis alérgica, dermatitis atópica y asma ${ }^{29,30}$. También puede asociarse a rinosinusitis, tos crónica, crup recurrente y ronquera $^{1}$.

\section{Diagnóstico}

Según los consensos actuales ${ }^{14,31}$, EoE se diagnostica cuando hay síntomas de disfunción esofágica y al menos 15 eosinófilos/hpf en la biopsia de mucosa esofágica y después de una evaluación exhaustiva de trastornos no EoE que pueden causar o contribuir potencialmente a la eosinofilia esofágica. La evidencia sugiere que los IBP están mejor clasificados como un tratamiento para la eosinofilia esofágica que puede deberse a la EoE, más que a un criterio diagnóstico ${ }^{13}$.

\section{Estudio endoscópico}

La EDA permite visualizar hallazgos que aumentan la probabilidad clínica de eosinofilia esofágica histológica y tomar biopsias que permitirán el diagnóstico de EoE. Por lo tanto, ante la sospecha clínica de EoE o en el estudio de disfagia es importante la solicitud de una EDA con biopsias escalonadas de esófago. Los hallazgos endoscópicos no son patognomónicos para EoE y es posible que no respondan de manera uniforme al tratamiento y los informes son variables dependiendo del observador ${ }^{31}$. Un metaanálisis ${ }^{32}$ estimó la frecuencia de los hallazgos endoscópicos: surcos lineales $48 \%$, anillos circulares $44 \%$, atenuación del patrón vascular subepitelial $41 \%$, pápulas blanquecinas (microabscesos de eosinófilos) 27\%, estenosis $21 \%$ y esófago de calibre pequeño $9 \%$ (Figuras 1A-C). El 17\% presentaba hallazgos normales, pero disminuyó a 7\% cuando el análisis estaba limitado a estudios prospectivos $(\mathrm{P}<0,05)$. Estos hallazgos varían según la edad. Los adultos presentan más anillos y estenosis, mientras que los niños tienen más probabilidades de tener placas blancas y disminución de la vascularización/ edema. Ningún hallazgo fue lo suficientemente sensible ni específico para utilizarse de manera aislada para diagnosticar EoE. 

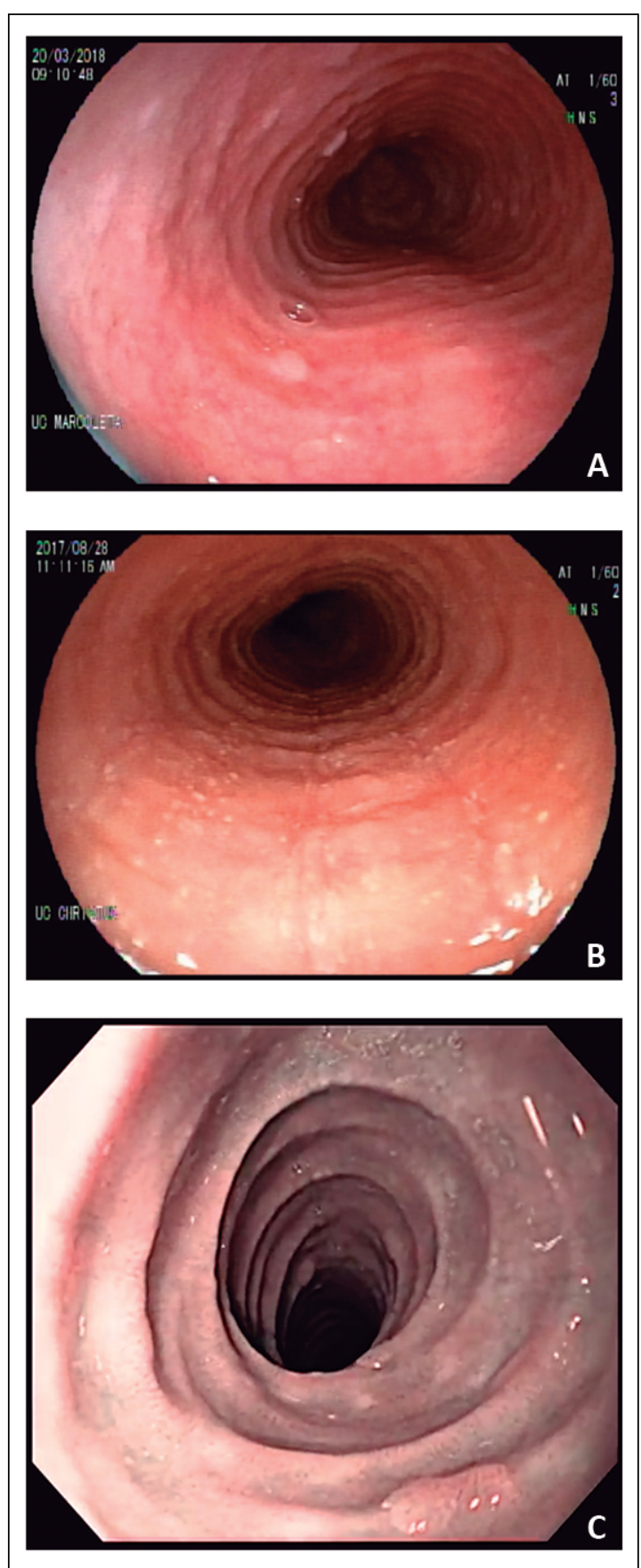

Figuras 1. Hallazgos endoscópicos de esofagitis eosinofílica. A: Pérdida del patrón vascular subepitelial, anillos circulares y pápulas blanquecinas (microabscesos de eosinófilos); B: surcos lineales, pápulas blanquecinas; C: Esófago evaluado con imagen de banda estrecha (NBI) marcadamente disminuido en calibre con numerosos anillos circulares ("esófago traquealizado") y pápulas blanquecinas. Unidad de Endoscopía Hospital Clínico UC-Christus.

\section{Histopatología}

Las biopsias esofágicas de pacientes con EoE muestran un aumento en el número de eosinófilos mayor a 15 eos/hpf, pueden formar microabscesos $y$ alinearse de forma paralela a la superficie luminal (Figura 2$)^{13}$. Los eosinófilos se reconocen fácilmente en secciones de tejido, fijadas en formalina, que se tiñen con hematoxilina y eosina, no se requieren tinciones especiales ${ }^{34}$. Varios estudios han demostrado que la sensibilidad de las biopsias para diagnosticar EoE aumenta mientras mayor sea el número de estas, sugiriendo que un buen número sería obtener 3 biopsias de esófago proximal y 3 de esófago distal (Figura 3) 35-37. En caso de presentar síntomas sugerentes de gastroenteritis eosinofílica (dolor abdominal, náuseas, vómitos, diarrea, baja de peso, ascitis) o anomalías visibles de la mucosa, se deben obtener biopsias de antro gástrico y duodeno, ya que, de ser diagnosticada gastroenteritis eosinofílica, cambiará el tratamiento ${ }^{7}$.

\section{Test de laboratorio}

Los estudios de laboratorio tienen utilidad limitada en la evaluación de pacientes con EoE, aunque hasta $40 \%$ de los pacientes con EoE tienen eosinofilia, generalmente es una elevación leve y no diagnóstica. Se pueden observar niveles elevados de inmunoglobulina E ( $\mathrm{IgE}$ ) hasta en $50 \%$ de los pacientes con EoE. Sin embargo, tanto la ausencia como la presencia de IgE elevada no descarta ni asegura el diagnóstico, respectivamente $^{38,39}$. Biomarcadores séricos están siendo estudiados para ayudar al diagnóstico y monitoreo no invasivo de EoE, pero ninguno ha sido aprobado para uso clínico. La determinación de IgE específica podría tener un rol en la evaluación de la reincorporación de los alimentos en aquellos pacientes sensibilizados, para evitar reacciones alérgicas alimentarias.

\section{Estudios radiológicos}

Los estudios radiológicos tienen un valor limitado en el diagnóstico de EoE. Estudios de contraste del tracto gastrointestinal superior no son los suficientemente sensibles como para captar los cambios sutiles de EoE, pero pueden ayudar 


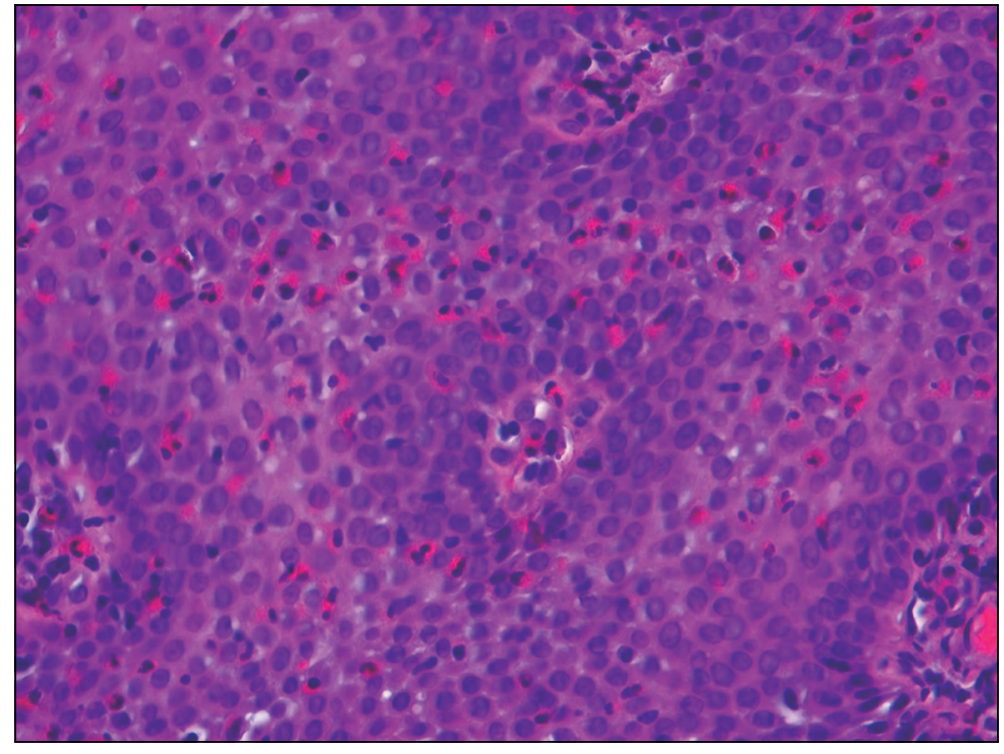

Figura 2. Imagen histopatológica de esofagitis eosinofílica demostrando abundante eosinofilia: > 15 eosinófilos por campo de aumento mayor. Tinción con hematoxilina-eosina. Dra. Javiera Torres, Departamento Anatomía Patológica, Pontificia Universidad Católica de Chile.

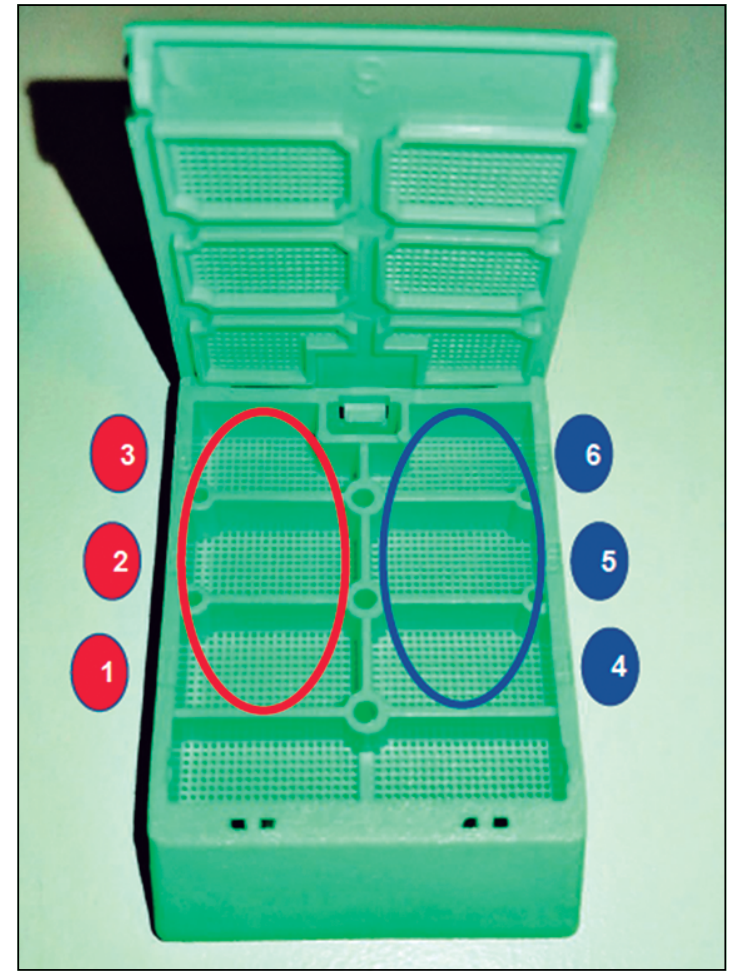

Figura 3. Protocolo Unidad de Endoscopía Hospital UC-Christus para toma de biopsias esofágicas en casos de disfagia/ impactación alimentaria. Muestras 1-3: Esófago proximal. Muestras 4-6: Esófago distal. a identificar otras patologías o estenosis esofágica por EoE, que puede ser extensa y difícil de valorar durante la $\mathrm{EDA}^{40,41}$.

\section{Tratamiento}

El tratamiento tiene como objetivos reducir los síntomas, mejorar la calidad de vida y prevenir fibrosis y remodelamiento del esófago, lo cual se asocia a formación de estenosis e impactación de alimentos ${ }^{42}$. Existen 3 enfoques principales de tratamiento para la EoE: dieta, drogas y dilatación (Figura 4). El tratamiento debería ser iniciado con IBP, un agente antiinflamatorio (corticoide tópico) o dieta de eliminación. La selección depende exclusivamente de la preferencia del paciente y médico tratante, porque ningún estudio comparativo ha mostrado que alguno de ellos sea superior a los otros. La dilatación está indicada en presencia de estenosis. El tratamiento requiere una evaluación y seguimiento multidisciplinario por gastroenterología, nutrición e inmunología.

\section{Dieta}

La EoE es una patología producida principalmente por antígenos alimentarios, en este sentido, la evidencia demuestra que la dieta de eliminación 


\section{Tratamiento - Inducción}

IBP doble dosis o corticoides tópicos alta dosis o dieta de eliminación*

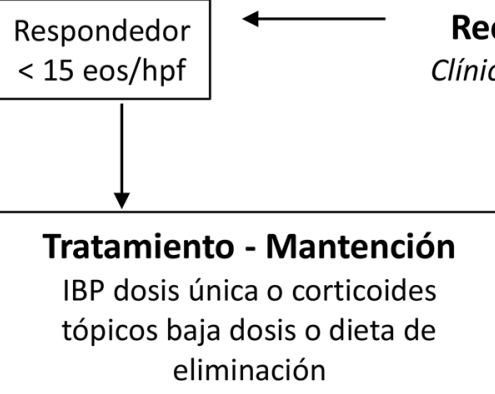

Si el paciente está con corticoides tópicos $\rightarrow$ Reinducción o dieta Si el paciente está con IBP $\rightarrow$ Cambiar a corticoides tópicos Si el paciente está con dieta $\rightarrow$ Cambiar a corticoides tópicos

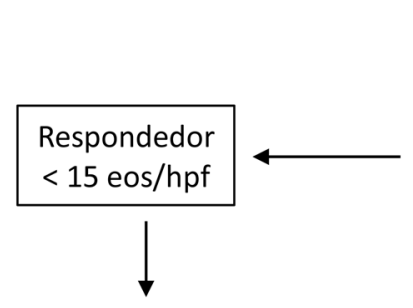

Reevaluación a 1 año

Clínica, endoscópica e histológica

\section{Reevaluación a 2-3 meses} Clínica, endoscópica e histológica

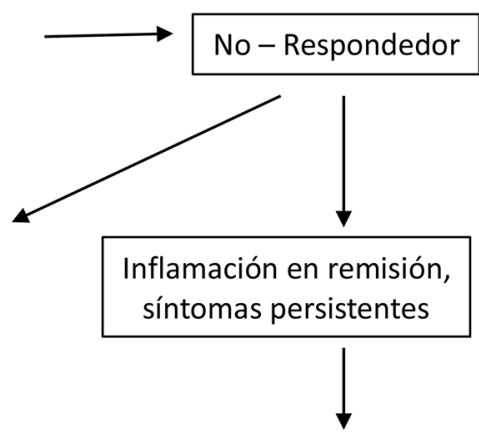

Estenosis $\rightarrow$ Dilatación endoscópica

Ausencia estenosis $\rightarrow$ Excluir otras enfermedades /

Reevaluar diagnóstico inicial

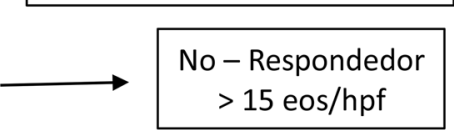

Derivar a un centro de experiencia

Considerar dieta elemental - Fármacos experimentales

Figura 4. Algoritmo de tratamiento de esofagitis eosinofílica. *El tratamiento debe comenzar con un IBP, corticoides tópicos o una dieta de eliminación. La selección del tratamiento dependerá la preferencia del paciente y del médico tratante, esto debido a que no existen estudios que hayan mostrado superioridad de uno sobre otro. IBP: Inhibidores de la bomba de protones. Eos/ hpf: Eosinófilos por campo de aumento mayor.

de los alimentos que gatillan la respuesta inflamatoria es un tratamiento usado en niños y adultos, especialmente luego de haber usado IBP ${ }^{43-47}$.

1) Dieta elemental: Todos los alimentos sólidos se reemplazan con una fórmula elemental, a base de aminoácidos. Su eficacia es alta sobre $90 \%$, su desventaja es el costo y la adherencia, ya que la monotonía de la dieta no favorece su seguimien- to a largo plazo, además, dificulta la posibilidad de identificar los alimentos que son capaces de inducir la respuesta, para iniciar el período de reintroducción del alimento. Actualmente no recomendada ${ }^{43,44,47}$.

2) Dieta de eliminación dirigida: Implica eliminación de alimentos basado en pruebas de alergia. $\mathrm{Su}$ eficacia es de $45 \%{ }^{47}$. La precisión diagnóstica 
puede ser un problema debido a resultados falsos positivos y negativos. Guías actuales recomiendan evitar esta estrategia.

3) Dieta de eliminación empírica: Consiste en la eliminación de alimentos que han demostrado ser potenciales alérgenos, como la leche de vaca, soya, trigo, huevo, palta o frutos secos y pescados o mariscos. La dieta de eliminación de seis alimentos (DESA) ha demostrado efecto positivo sobre niños y adultos ${ }^{45,46}$. Un estudio retrospectivo demostró que la intervención con DESA en niños mejora la respuesta inflamatoria, clínica e histológica en $74 \%{ }^{46}$ y en adultos la respuesta es similar ${ }^{47}$. En un análisis de 702 pacientes en 11 estudios se vio que, de estos seis grupos de alimentos, los que presentaban, en promedio, mayor respuesta antigénica eran leche de vaca $(66 \%)$ y trigo $(27 \%)$, luego huevos $(24 \%)$, soya $(12 \%)$ y maní $(4 \%)^{48}$.

Esta dieta debe ser seguida de reintroducción de alimentos de forma individual, secuencial, guiada y evaluada histológicamente. La reintroducción se debe hacer de a un alimento, luego de seis semanas con DESA y haber demostrado una favorable respuesta histológica $(<15$ eos/ hpf, buena tolerancia). Si se presenta $>15$ eos/ hpf, se considera mala tolerancia y se identifica a ese alimento como desencadenante, en ese caso se elimina el alimento de la dieta, para luego seguir la reintroducción de otros alimentos. La respuesta favorable y sostenida en el tiempo se considera cuando hay ausencia de eosinófilos en muestras de biopsia al primer, segundo y tercer años luego de terminada la reintroducción de alimentos, además de ausencia de síntomas relacionados con EoE y sin necesidad de tratamiento adicional de fárma$\cos ^{49}$. Estas dietas requieren evaluación periódica para evitar déficit calórico, de macronutrientes (proteína) y de micronutrientes (calcio, zinc, magnesio, ácido fólico, niacina y vitamina B12 y D). Es importante considerar la adherencia a la dieta, déficit nutricional del paciente e impacto social de estas dietas. Recientemente, la estrategia más razonable de eliminación de alimentos es el "step up" 2-4-6, es decir, comenzar a eliminar los dos alimentos que han demostrado mayor respuesta clínica e histológica (leche y trigo), evaluar su impacto, y luego según respuesta eliminar 4 o 6 alimentos. La remisión de síntomas con 2-4-6 alimentos resultó en $43 \%, 60 \%$ y $79 \%$, respectivamente. Evita restricciones innecesarias, ahorrando endoscopías y acortando el proceso diagnóstico ${ }^{50}$.

\section{Inhibidores de la bomba de protones}

Una revisión sistemática demostró remisión histológica con IBP en adultos en 50,5\% y mejora sintomática en $60,8 \%{ }^{51}$. Sin embargo, la evidencia es de baja calidad, resultados heterogéneos y sesgo de publicación a favor de la respuesta a IBP. Otro estudio prospectivo, en cohorte pediátrica, mostró que $78 \%$ de los respondedores a IBP mantienen remisión clínico-patológica a 1 año de seguimiento $^{52}$. Un estudio multicéntrico de seguimiento a largo plazo en 75 adultos con PPI-REE demostró que la suspensión de tratamiento implica recaída sintomática o histológica ${ }^{53}$.

Debido a su bajo costo, buen perfil de seguridad, conveniencia y gran cantidad de literatura que describe un grupo de EoE respondedor a IBP, actualmente se considera a los IBP como un tratamiento de primera línea ${ }^{13}$. No existen datos sobre su efecto sobre fibrosis y hay datos limitados de seguimiento a largo plazo (no mayores a 1 año $)^{51}$.

En niños se recomienda $1 \mathrm{mg} / \mathrm{kg}$ por dosis 2 veces al día de esomeprazol y en adultos doble dosis (20 mg cada dosis) por al menos 8 semanas antes de evaluar respuesta ${ }^{52}$. No se documentan diferencias significativas respecto del tipo de IBP evaluado $^{51}$. En el seguimiento histológico endoscópico, si persisten los síntomas o $>15$ eos/ hpf, se recomienda cambio de terapia a dieta o corticoides; mientras que si hay $<15$ eos/hpf, se recomienda reducir la dosis y mantener IBP una vez al día antes de un nuevo control ${ }^{54}$.

\section{Corticoides}

Los corticoides tópicos, con efecto directo sobre mucosa, han logrado buena eficacia clínicopatológica y efecto antifibrótico ${ }^{42}$, minimizando los efectos adversos de corticoides sistémicos (no se encuentran recomendados $)^{55}$. Un estudio de distribución aleatoria controlado utilizó suspensión de budesonida oral, no disponible en Chile actualmente, demostrando mejora significativa en puntuación de síntomas de disfagia, hallazgos histológicos y endoscópicos ${ }^{56}$. Otro estudio controlado y aleatorio evaluó budesonida en tableta, suspensión viscosa de budesonida o placebo. Se encontró remisión histológica y mejora de score endoscópico en ambos grupos de budesonida vs placebo, con 2 semanas de terapia ${ }^{57}$. Un análisis de cintigrafía demostró que la formulación viscosa tenía contacto prolongado con la mucosa esofágica 
en comparación a nebulizada ${ }^{58}$. Otro estudio mostró que pacientes tratados con budesonida viscosa oral obtuvieron remisión histológica $(75 \%)$ más a menudo que los tratados con fluticasona en aerosol ingerida $(40 \%)^{59}$, lo que no ha sido replicado en estudios recientes, donde se obtuvieron respuestas similares con ambos fármacos ${ }^{60}$. La dosis recomendada de budesonida en suspensión viscosa oral es de $1 \mathrm{mg} /$ día para niños $<10$ años y $2 \mathrm{mg} /$ día para niños mayores y adultos. Esta se puede preparar al mezclar con sucralosa ( $1 \mathrm{~g}$ sucralosa por cada $1 \mathrm{mg}$ de budesonida $)^{61}$. La dosis de fluticasona en niños varía de 88 a $440 \mathrm{mcg} /$ día en dos dosis, mientras que en adultos varía desde 880 a $1.760 \mathrm{mcg} /$ día en dos dosis ${ }^{62}$. Se debe evitar la ingestión de alimentos y líquidos por al menos 30 min después del medicamento ${ }^{54}$. Los corticoides tópicos tienen un perfil de seguridad favorable, sin efectos serios reportados. La candidiasis esofágica es observada hasta en $10 \%{ }^{63}$. A largo plazo, la terapia con corticoides tópicos reportó una pérdida de respuesta en $50 \%$ pacientes con EoE en 18,5 meses y $75 \%$ en 29,6 meses $^{64}$. Otro estudio con budesonida demostró pérdida de respuesta histológica tras 1 año de seguimiento ${ }^{65}$. Un estudio de budesonida en suspensión oral encontró 58\% de pérdida de respuesta histológica a las 24 semanas de seguimiento ${ }^{66}$. Los tres estudios atribuyen parte de la pérdida de respuesta a la reducción de dosis durante la fase de mantención, pero se requiere más información para determinar si existe desarrollo de tolerancia. Actualmente se desarrolla una presentación de budesonida bucodispersable con buena respuesta en estudios iniciales, como alterativa prometedora para simplificar el tratamiento y mejorar la adherencia ${ }^{67}$.

\section{Terapias biológicas}

Varios agentes biológicos están siendo estudiados para uso en EoE, tales como mepolizumab, reslizumab, QAX576, omalizumab, dupilumab. De ser eficaces, sus ventajas incluirían evitar el uso diario de esteroides. Sus efectos sistémicos pueden beneficiar a múltiples formas de enfermedad alérgica en individuos altamente atópicos, aplicación para pacientes refractarios a IBP/corticoides y capacidad de remodelación esofágica. Sin embargo, su costo es alto, su disponibilidad es baja y aún existen datos limitados sobre su eficacia a corto y largo plazo, por lo que no se consideran parte de la terapia estándar ${ }^{68}$.

\section{Dilatación esofágica}

La dilatación esofágica se recomienda solo cuando se producen estenosis o cambios fibroestenóticos. Un metaanálisis revisó 845 pacientes con EoE sometidos a 1.820 dilataciones esofágicas. Los síntomas mejoraron en más de $95 \%$ de los pacientes. Las medidas promedio predilatación fueron 9,9 $\mathrm{mm}$ y postdilatación $16 \mathrm{~mm}$. En promedio, se sometieron a 3 dilataciones (rango 1-35). Dentro de las complicaciones destacan perforación $(0,38 \%)$, hemorragia $(0,05 \%)$ y hospitalización $(0,67 \%)$, sin muertes ${ }^{69}$. Puede existir dolor torácico posterior al procedimiento transitorio. No tiene influencia sobre la inflamación subyacente y fibrosis ${ }^{42}$.

\section{Pronóstico}

El conocimiento sobre la historia natural de EoE es limitado y en muchos aspectos incompletamente definido. Los datos disponibles no sugieren que EoE afecte la expectativa de vida o asociación con cáncer, pero existe la preocupación de que la inflamación crónica no controlada puede resultar en un daño irreversible estructural esofágico, lo que lleva a fibrosis, estenosis y función esofágica alterada. Un incremento en el tiempo de EoE no tratada estaría fuertemente asociado con esteno$\mathrm{sis}^{70}$. En suma, su diagnóstico y tratamiento precoz es clave para evitar o posponer sus complicaciones, tales como estenosis y disfunción esofágica grave.

\section{Referencias}

1. Kumar S, Choi S, Gupta SK. Eosinophilic Esophagitis-A Primer for Otolaryngologists. JAMA Otolaryngol Head Neck Surg 2019; 145 (4): 373-80.

2. Liacouras CA, Furuta GT, Hirano I, Atkins D, Attwood SE, Bonis PA, et al. Eosinophilic esophagitis: updated consensus recommendations for children and adults. J Allergy Clin Immunol 2011; 128 (1): 3-20.

3. Attwood SE, Smyrk TC, Demeester TR, Jones JB. Esophageal eosinophilia with dysphagia. A distinct clinicopathologic syndrome. Dig Dis Sci 1993; 38 (1): 109-16.

4. Giriens B, Yan P, Safroneeva E, Zwahlen M, Reinhard A, Nydegger A, et al. Escalating incidence of eosinophilic esophagitis in Canton of Vaud, Switzerland, 1993-2013: a population-based study. Allergy 2015; 70 (12): 1633-9. 
5. Larsson H, Bergman K, Finizia C, Johansson L, Bove M, Bergquist H. Dysphagia and health-related quality of life in patients with eosinophilic esophagitis: a long-term follow-up. Eur Arch Otorhinolaryngol 2015; 272 (12): 3833-9.

6. Furuta GT, Katzka DA. Eosinophilic Esophagitis. N Engl J Med 2015; 373 (17): 1640-8.

7. Dellon ES, Gonsalves N, Hirano I, Furuta GT, Liacouras CA, Katzka DA; American College of Gastroenterology. ACG clinical guideline: Evidenced based approach to the diagnosis and management of esophageal eosinophilia and eosinophilic esophagitis (EoE). Am J Gastroenterol 2013; 108 (5): 679-92.

8. Hirano I. How to Approach a Patient With Eosinophilic Esophagitis. Gastroenterology 2018; 155 (3): 601-6.

9. Rank MA, Sharaf RN, Furuta GT, Aceves SS, Greenhawt $\mathrm{M}$, Spergel JM et al. Technical review on the management of eosinophilic esophagitis: a report from the AGA institute and joint task force in allergy-immunology practice parametrs. Gastroenterology 2020; 158(6): 1789-810.

10. Moawad FJ, Schoepfer AM, Safroneeva E, Ally MR, Chen YJ, Maydonovitch CL, et al. Eosinophilic oesophagitis and proton pump inhibitor-responsive oesophageal eosinophilia have similar clinical, endoscopic and histological findings. Aliment Pharmacol Ther 2014; 39 (6): 603-8.

11. Vázquez-Elizondo G, Ngamruengphong S, Khrisna M, DeVault KR, Talley NJ, Achem SR. The outcome of patients with oesophageal eosinophilic infiltration after an eight-week trial of a proton pump inhibitor. Aliment Pharmacol Ther 2013; 38 (10): 1312-9.

12. Ishimura N, Kinoshita Y. Eosinophilic esophagitis in Japan: Focus on response to acid suppressive therapy. J Gastroenterol Hepatol 2018; 33 (5): 1016-22.

13. Dellon E, Liacouras CA, Molina-Infante J, Furuta GT, Spergel JM, Zevit N, et al. Updated International Consensus Diagnostic Criteria for Eosinophilic Esophagitis: Proceedings of the AGREE Conference. Gastroenterology 2018; 155 (4): 1022-33.

14. Lucendo AJ, Molina-Infante J, Arias Á, von Arnim U, Bredenoord AJ, Bussmann C, et al. Guidelines on eosinophilic esophagitis: evidence-based statements and recommendations for diagnosis and management in children and adults. United European Gastroenterol J 2017; 5 (3): 335-58.

15. Moawad FJ. Eosinophilic Esophagitis: Incidence and Prevalence. Gastrointest Endosc Clin N Am. 2018; 28 (1): $15-25$.

16. García-Compeán D, González-González JA, González-Moreno EI, Maldonado-Garza HJ. La esofagitis eosinofílica. ¿El Norte contra el Sur? Enfoque mecanicista bio-económico-social e implicaciones clínicas. Rev Gastroenterol Mex 2017; 82 (4): 328-36.

17. González F CG, Torres J, Molina U R, Harris PR. Esofagitis eosinofílica en niños: Características clínicas y endoscópicas. Rev Med Chile 2009; 137 (5): 666-71.

18. Pavez C, Silva J, Díaz R. Esofagitis eosinofílica. A propósito de un caso clínico. Gastr Latinoam 2006; 17 (1): 73-8.

19. Planzer M. Esofagitis eosinofílica. Gastr Latinoam 2007; 18 (1): 136-40.

20. Dellon ES, Jensen ET, Martin CF, Shaheen NJ, Kappelman MD. Prevalence of eosinophilic esophagitis in the United States. Clin Gastroenterol Hepatol 2014; 12 (4): 589-96.

21. Prasad GA, Alexander JA, Schleck CD, Zinsmeister AR, Smyrk TC, Elias RM, et al. Epidemiology of eosinophilic esophagitis over three decades in Olmsted County, Minnesota. Clin Gastroenterol Hepatol 2009; 7 (10): 1055-61.

22. Veerappan GR, Perry JL, Duncan TJ, Baker TP, Maydonovitch C, Lake JM, et al. Prevalence of eosinophilic esophagitis in an adult population undergoing upper endoscopy: a prospective study. Clin Gastroenterol Hepatol 2009; 7 (4): 420-6.

23. Sealock RJ, Kramer JR, Verstovsek G, Richardson P, Rugge M, Parente P, et al. The prevalence of oesophageal eosinophilia and eosinophilic oesophagitis: a prospective study in unselected patients presenting to endoscopy. Aliment Pharmacol Ther 2013; 37 (8): 825-32.

24. Ricker J, McNear S, Cassidy T, Plott E, Arnold H, Kendall B, et al. Routine screening for eosinophilic esophagitis in patients presenting with dysphagia. Therap Adv Gastroenterol 2011; 4 (1): 27-35.

25. Lenz CJ, Leggett C, Katzka DA, Larson JJ, Enders FT, Alexander JA. Food impaction: etiology over 35 years and association with eosinophilic esophagitis. Dis Esophagus 2019; 32 (4) 1-7.

26. Shaheen NJ, Mukkada V, Eichinger CS, Schofield H, Todorova L, Falk GW. Natural history of eosinophilic esophagitis: a systematic review of epidemiology and disease course. Dis Esophagus 2018; 31 (8): 1-14.

27. Clayton F, Fang JC, Gleich GJ, Lucendo AJ, Olalla JM, Vinson LA, et al. Eosinophilic esophagitis in adults is associated with IgG4 and not mediated by IgE. Gastroenterology 2014;147 (3): 602-9.

28. Chen JW, Kao JY. Eosinophilic esophagitis: update on management and controversies. BMJ. 2017; 359: j4482.

29. Roy-Ghanta S, Larosa DF, Katzka DA. Atopic characteristics of adult patients with eosinophilic esophagitis. Clin Gastroenterol Hepatol 2008; 6 (5): 531-5. 
30. Durrani SR, Mukkada VA, Guilbert TW. Eosinophilic Esophagitis: an Important Comorbid Condition of Asthma?. Clin Rev Allergy Immunol. 2018; 55 (1): 56-64.

31. Spergel JM, Dellon ES, Liacouras CA, Hirano I, Molina-Infante J, Bredenoord AJ, et al. Summary of the updated international consensus diagnostic criteria for eosinophilic esophagitis. Ann Allergy Asthma Immunol 2018; 121 (3): 281-4.

32. Dellon ES. Do you see what I see? Towards standardized reporting of endoscopic findings in eosinophilic esophagitis. Endoscopy 2014; 46 (12): 1043-5.

33. Kim HP, Vance RB, Shaheen NJ, Dellon ES. The Prevalence and Diagnostic Utility of Endoscopic Features of Eosinophilic Esophagitis: A Meta-Analysis. Clin Gastroenterol Hepatol 2012;10 (9): 988-96.

34. Collins MH. Histopathology of eosinophilic esophagitis. Dig Dis 2014; 32 (1-2): 68-73.

35. Gonsalves N, Policarpio-Nicolas M, Zhang Q, Rao MS, Hirano I. Histopathologic variability and endoscopic correlates in adults with eosinophilic esophagitis. Gastrointest Endosc 2006; 64 (3): 313-9.

36. Shah A, Kagalwalla AF, Gonsalves N, Melin-Aldana H, Li BU, Hirano I. Histopathologic variability in children with eosinophilic esophagitis. Am J Gastroenterol 2009; 104 (3): 716-21.

37. Nielsen JA, Lager DJ, Lewin M, Rendon G, Roberts CA. The optimal number of biopsy fragments to establish a morphologic diagnosis of eosinophilic esophagitis. Am J Gastroenterol. 2014; 109 (4): 515-20.

38. Ishimura N, Furuta K, Sato S, Ishihara S, Kinoshita Y. Limited role of allergy testing in patients with eosinophilic gastrointestinal disorders. J Gastroenterol Hepatol 2013; 28 (8): 1306-13.

39. Hiremath G, Gupta SK. Promising Modalities to Identify and Monitor Eosinophilic Esophagitis. Clin Gastroenterol Hepatol 2017; 15 (11): 1655-64.

40. Gentile N, Katzka D, Ravi K, Trenkner S, Enders F, Killian J, et al. Oesophageal narrowing is common and frequently under-appreciated at endoscopy in patients with oesophageal eosinophilia. Aliment Pharmacol Ther 2014; 40 (11-12): 1333-40.

41. Menard-Katcher C, Swerdlow MP, Mehta P, Furuta GT, Fenton LZ. Contribution of Esophagram to the Evaluation of Complicated Pediatric Eosinophilic Esophagitis. J Pediatr Gastroenterol Nutr. 2015; 61 (5): 541-6.

42. Schoepfer AM, Straumann A, Safroneeva E. Pharmacologic Treatment of Eosinophilic Esophagitis: An Update. Gastrointest Endosc Clin N Am 2018; 28 (1): 77-88.

43. Peterson KA, Byrne KR, Vinson LA, Ying J, Boynton KK, Fang JC, et al. Elemental diet induces histologic response in adult eosinophilic esophagitis. Am J Gastroenterol 2013; 108 (5): 759-66.

44. Vashi R, Hirano I. Diet therapy for eosinophilic esophagitis: when, why and how. Curr Opin Gastroenterol 2013; 29 (4): 407-15.

45. Gonsalves N, Yang GY, Doerfler B, Ritz S, Ditto AM, Hirano. Elimination diet effectively treats eosinophilic esophagitis in adults; food reintroduction identifies causative factors. Gastroenterology 2012; 142 (7): 1451-9.

46. Kagalwalla AF, Sentongo TA, Ritz S, Hess T, Nelson SP, Emerick KM, et al. Effect of six-food elimination diet on clinical and histologic outcomes in eosinophilic esophagitis. Clin Gastroenterol Hepatol 2006; 4 (9): 1097-102.

47. Arias A, González-Cervera J, Tenias JM, Lucendo AJ. Efficacy of dietary interventions for inducing histologic remission in patients with eosinophilic esophagitis: a systematic review and meta-analysis. Gastroenterology 2014; 146 (7): 1639-48.

48. Spergel J, Aceves SS. Allergic components of eosinophilic esophagitis. J Allergy Clin Immunol 2018; 142 (1): 1-8.

49. Lucendo AJ, Arias Á, González-Cervera J, Yagüe-Compadre JL, Guagnozzi D, Angueira T, et al. Empiric 6-food elimination diet induced and maintained prolonged remission in patients with adult eosinophilic esophagitis: a prospective study on the food cause of the disease. J Allergy Clin Immunol 2013; 131 (3): 797-804.

50. Molina-Infante J, Arias Á, Alcedo J, García-Romero $\mathrm{R}$, Casabona-Frances S, Prieto-García A, et al. Step-up empiric elimination diet for pediatric and adult eosinophilic esophagitis: The 2-4-6 study. J Allergy Clin Immunol 2018; 141(4): 1365-72.

51. Lucendo AJ, Arias Á, Molina-Infante J. Efficacy of Proton Pump Inhibitor Drugs for Inducing Clinical and Histologic Remission in Patients With Symptomatic Esophageal Eosinophilia: A Systematic Review and Meta-Analysis. Clin Gastroenterol Hepatol 2016; 14 (1): $13-22$.

52. Gutiérrez-Junquera C, Fernández-Fernández S, Cilleruelo ML, Rayo A, Echeverría L, Quevedo S, et al. High Prevalence of Response to Proton-pump Inhibitor Treatment in Children With Esophageal Eosinophilia. J Pediatr Gastroenterol Nut 2016; 62 (5): 704-10.

53. Molina-Infante J, Rodríguez-Sánchez J, Martinek J, van Rhijn BD, Krajciova J, Rivas MD, et al. Long-Term Loss of Response in Proton Pump Inhibitor-Responsive Esophageal Eosinophilia Is Uncommon and Influenced by CYP2C19 Genotype and Rhinoconjunctivitis. Am J Gastroenterol 2015; 110 (11): 1567-75.

54. Ferreira CT, Vieira MC, Furuta GT, Barros FCLF, Che- 
hade M. Eosinophilic esophagitis-Where are we today?. J Pediatr 2019; 95 (3): 275-81.

55. Patel RV, Hirano I. New Developments in the Diagnosis, Therapy, and Monitoring of Eosinophilic Esophagitis. Curr Treat Options Gastroenterol 2018; 16 (1): 15-26.

56. Dellon ES, Katzka DA, Collins MH, Hamdani M, Gupta SK, Hirano I, et al. Budesonide Oral Suspension Improves Symptomatic, Endoscopic, and Histologic Parameters Compared With Placebo in Patients With Eosinophilic Esophagitis. Gastroenterology 2017; 152 (4): 776-86.

57. Miehlke S, Hruz P, Vieth M, Bussmann C, von Arnim $\mathrm{U}$, Bajbouj $\mathrm{M}$, et al. A randomised, double-blind trial comparing budesonide formulations and dosages for short-term treatment of eosinophilic oesophagitis. Gut 2015; 65 (3): 390-9.

58. Dellon ES, Sheikh A, Speck O, Woodward K, Whitlow $\mathrm{AB}$, Hores JM, et al. Viscous topical is more effective than nebulized steroid therapy for patients with eosinophilic esophagitis. Gastroenterology 2012; 143 (2): 321-4.

59. Fable J, Fernández M, Goodine S, Lerer T, Sayej W. Retrospective Comparison of Fluticasone Propionate and Oral Viscous Budesonide in Children With Eosinophilic Esophagitis. J Pediatr Gastroenterol Nut 2018; 66 (1): 26-32.

60. Dellon ES, Woosley JT, Arrington A, McGee SJ, Covington J, Moist SE, et al. Efficacy of Budesonide vs Fluticasone for Initial Treatment of Eosinophilic Esophagitis in a Randomized Controlled Trial. Gastroenterology. 2019; 157 (1): 65-73.

61. Dohil R, Newbury R, Fox L, Bastian J, Aceves S. Oral viscous budesonide is effective in children with eosinophilic esophagitis in a randomized, placebo-controlled trial. Gastroenterology 2010; 139 (2): 418-29.

62. Schaefer ET, Fitzgerald JF, Molleston JP, Croffie JM, Pfefferkorn MD, Corkins MR, et al. Comparison of Oral Prednisone and Topical Fluticasone in the Treatment of Eosinophilic Esophagitis: A Randomized Trial in Chil- dren. Clin Gastroenterol Hepatol 2008; 6 (2): 165-73.

63. Murali AR, Gupta A, Attar BM, Ravi V, Koduru P. Topical steroids in eosinophilic esophagitis: Systematic review and meta-analysis of placebo-controlled randomized clinical trials. J Gastroenterol Hepatol 2016; 31 (6): 1111-9.

64. Eluri S, Runge TM, Hansen J, Kochar B, Reed CC, Robey BS, et al. Diminishing Effectiveness of LongTerm Maintenance Topical Steroid Therapy in PPI Non-Responsive Eosinophilic Esophagitis. Clin Transl Gastroenterol 2017; 8 (6): e97.

65. Straumann A, Conus S, Degen L, Frei C, Bussmann C, Beglinger $\mathrm{C}$, et al. Long-term budesonide maintenance treatment is partially effective for patients with eosinophilic esophagitis. Clin Gastroenterol Hepatol 2011; 9 (5): 400-9.

66. Dellon ES, Katzka DA, Collins MH, Hamdani M, Gupta SK, Hirano I. Safety and Efficacy of Oral Budesonide Suspension for Maintenance Therapy in Eosinophilic Esophagitis: Results From a Prospective Open-Label Study of Adolescents and Adults. Gastroenterology 2016; 150 (4): S188.

67. Lucendo AJ, Miehlke S, Schlag C, Vieth M, von Arnim U, Molina-Infante J, et al. Efficacy of Budesonide Orodispersible Tablets as Induction Therapy for Eosinophilic Esophagitis in a Randomized Placebo-controlled Trial. Gastroenterology 2019; 157 (1): 74-86.

68. Ko E, Chehade M. Biological Therapies for Eosinophilic Esophagitis: Where Do We Stand?. Clin Rev Allergy Immunol 2018; 55 (2): 205-16.

69. Moawad FJ, Molina-Infante J, Lucendo AJ, Cantrell SE, Tmanova L, Douglas KM. Systematic review with meta-analysis: endoscopic dilation is highly effective and safe in children and adults with eosinophilic oesophagitis. Aliment Pharmacol Ther 2017; 46 (2): 96-105.

70. Schoepfer AM, Safroneeva E, Bussmann C, Kuchen T, Portmann S, Simon HU, et al. Delay in diagnosis of eosinophilic esophagitis increases risk for stricture formation in a time-dependent manner. Gastroenterology 2013; 145 (6): 1230-6. 